\title{
CAN LISTENING TO MUSIC MAKE YOU TYPE BETTER? THE EFFECT OF MUSIC STYLE, VOCALS AND VOLUME ON TYPING PERFORMANCE
}

\author{
Anna Bramwell-Dicks \\ Department of Theatre, Film and Television, \\ Department of Computer Science \\ University of York, UK \\ anna.bramwell-dickseyork.ac.uk
}

\author{
Helen Petrie and Alistair Edwards \\ Department of Computer Science \\ University of York, UK \\ helen.petriedyork.ac.uk \\ alistair.edwardseyork.ac.uk
}

\begin{abstract}
Music psychologists have frequently shown that music affects people's behaviour. Applying this concept to work-related computing tasks has the potential to lead to improvements in a person's productivity, efficiency and effectiveness. This paper presents two quantitative experiments exploring whether transcription typing performance is affected when hearing a music accompaniment that includes vocals. The first experiment showed that classifying the typists as either slow or fast ability is important as there were significant interaction effects once this between group factor was included, with the accuracy of fast typists reduced when the music contained vocals. In the second experiment, a Dutch transcription typing task was added to manipulate task difficulty and the volume of playback was included as a between groups independent variable. When typing in Dutch the fast typists' speed was reduced with louder music. When typing in English the volume of music had little effect on typing speed for either the fast or slow typists. The fast typists achieved lower speeds when the loud volume music contained vocals, but with low volume music the inclusion of vocals in the background music did not have a noticeable affect on typing speed. The presence of vocals in the music reduced the accuracy of the text entry across the whole sample. Overall, these experiments show that the presence of vocals in background music reduces typing performance, but that we might be able to exploit instrumental music to improve performance in tasks involving typing with either low or high volume music.
\end{abstract}

\section{INTRODUCTION}

Hearing music can affects people's behaviour. From the speed of drinking a can of soda [1], to the type of wine bought in a supermarket [2], to children's performance in arithmetic tasks [3], there is substantial empirical evidence that hearing music affects what people do and how well they do it. Our research aims to identify whether we can exploit music to positively influence people while they are working.

Many people spend a large proportion of their working lives using a computer. A study in 2007 collected objective data of computer-use at work across 95 organisations from Europe, North America and Australasia [4]. Highest levels of computer use were Commercial 4.0 International License. The full terms of the License are available at http://creativecommons.org/licenses/by-nc/4.0 identified in the UK, where over a 4 week period employees spent an average of 16.8 hours per week using a computer, i.e. approximately $40 \%$ of their working week. Given that people spend a large proportion of their working hours using a computer, we ask whether hearing music while interacting with a computer can positively affect performance in work-related computing activities?

Kallinen [5] showed that the speed of reading news stories on mobile devices while in a noisy café was affected by the tempo of the background music. Significantly faster reading speeds were achieved when listening to music with a higher tempo. Although a café is not a typical work environment and reading the news on a mobile device is not a typical work task, this result shows there is potential to exploit the impact of particular parameters of music to improve performance in work-related tasks. This outcome leads to a refinement of our research aims - we want to identify how different parameters of music affect people's performance with workrelated computing tasks.

Typing is a fundamental method used to interact with a computer. In 1937, Jensen investigated how hearing music affected the transcription typing performance of skilled typists [6]. The task required participants to copy text presented visually using a typewriter. The skilled typists made significantly more errors when listening to Jazz music than without music, or when accompanied by slow, melancholy Dirge music. Typing speeds were significantly slower with a Dirge music accompaniment than with Jazz music or without music. Jensen made no attempt to explain why Jazz increased error rates, or why Dirge music decreased typing speed. There does not seem to have been any follow up work to Jensen's experiment, so it is interesting investigate the impact of music on typing performance further, and within a modern context.

In this paper, we present two experiments investigating the effect of different parameters of music on transcription typing speed and accuracy. Although transcription typing is not a typical workrelated computing task, it is clearly-defined allowing us to retain tight control of the experiment, ensuring construct validity. The first experiment focuses on the impact of the presence or absence of vocals on typing performance, using two pieces of Rock music from different styles. The second experiment considers if the volume of the music is a significant factor affecting performance, and how the difficulty of the task mediates the influence of music.

\section{VOCALS AND MUSIC STYLE}

Shaffer [7] performed a number of experiments with a single, skilled typist, investigating how different verbal stimuli affect tran- 
scription typing performance. This typist was able to maintain high levels of performance when copying from a visual source whilst reciting nursery rhymes. Further, the typist was able to transcribe material that was presented aurally through headphones in one ear, while concurrently repeating words heard through the other ear. For this skilled typist, typing performance was maintained in the presence of potentially conflicting verbal material. But does this outcome extend to being able to perform transcription typing while hearing music containing vocals? And would less skilled typists be affected in a similar way?

\subsection{Method}

The aim of this first experiment is to investigate whether the presence of vocals in a piece of music affects transcription typing performance with two pieces of Rock music with different styles. Rationally, one might expect that hearing one verbal source whilst copying different visually presented verbal material would be a difficult task which compromised performance. But, this argument contradicts the outcome from Shaffer's work which has been influential in the typing literature (e.g. in [8]) and we have identified no other empirical evidence to support this hypothesis.

\subsubsection{Experimental Design and Hypotheses}

Two experimental design paradigms were used. The first was a 1 by 5 design with a single independent variable (IV) for music with 5 levels (Alt Rock With Vocals, Alt Rock Without Vocals, Pop Rock With Vocals, Pop Rock Without Vocals and Without Music). This design paradigm focuses on the impact of the pieces of music as a whole and permits inclusion of a Without Music condition. The second 2 by 2 design paradigm focuses on manipulating the presence of vocals (2 levels - with and without vocals) and the style of the music (2 levels - Alt Rock and Pop Rock).

Typing speed and accuracy were measured as dependent variables (DVs). The number of transcribed characters was established and uncorrected errors counted using the Levenshtein Minimum String Distance algorithm. Measures of Characters per Minute (CPM) for speed, and Error Rate for accuracy were calculated using and (1) and (2).

$$
\begin{aligned}
\mathrm{CPM} & =\frac{\text { Number of Characters }}{\text { Length of Task (in minutes) }} \\
\text { Error Rate } & =\frac{\text { Number of Errors }}{\text { Number of Characters }} * 100 \%
\end{aligned}
$$

\subsubsection{Participants}

The participants were recruited ( 22 male, 6 female) via an advert sent to University mailing lists, which stated that participants must be native English speakers, should not be dyslexic or have a hearing disability. Participants were aged between 18 and 44, 53\% aged 18 to 24. Twenty-two participants were studying Computer Science (11 undergraduate and $11 \mathrm{PhD}$ ), with 4 Social Science $\mathrm{PhD}$ students and 2 professional researchers from the Humanities. All participants received a $£ 10$ Amazon voucher.

\subsubsection{Materials and Environment}

The experiment took place in a quiet usability laboratory on the University campus. The room contained 1 desktop computer running Windows XP which the participant used. The typing tasks were hosted on a bespoke website accessed through the FireFox browser with spellcheck disabled. The website displayed two text boxes side by side. The left hand text box was non-editable and displayed the text to be copied. The participants entered their transcription in the right hand text box, the contents of which was saved on the webserver once the task had been completed. The experimenter used a MacBook Pro to control the music playback via Audacity connected to a pair of Philips SPA 22102.0 speakers.

Two pieces of Rock music were used in this experiment, both taken from Cambridge Music Technology website (http://www.cambridge-mt.com/). The first song, "Atrophy" by The Doppler Shift, was described as Alt Rock style, the second, "Big Dummy Shake" by Moosmusic is Pop Rock. For the experiment to have strong internal validity, the two variations of the music stimulus needed to be the same with the exception of the presence of vocals. Multi-track recordings were mixed down into two separate versions of each song. One version included all the vocal tracks while the other was a mix of only the instrumental tracks. It was also important that the participants were equally familiar or unfamiliar with the music. None of the participants could recall hearing either song prior to the experiment.

There were 5 conditions in the experiment. The order of presentation of music style was alternated so that the participants did not hear the two Alt Rock or Pop Rock conditions back to back. The style of music and inclusion of vocals were both counterbalanced to avoid learning and fatigue effects. The position of the Without Music condition was also systematically varied. Five passages from different chapters of The Outlaw of Torn (Edgar Rice Burroughs) were displayed to participants as the text for transcription. The order of presentation of the passages was varied to avoid connections between experiment condition and each text passage.

\subsubsection{Procedure}

The experimenter began by telling the participants that they would complete a number of transcription typing tasks while listening to different pieces of music to see how the music affected their performance. They were told they could correct any errors but that they should only use the keyboard and not the mouse and that they should type as naturally as possible. The participants completed a 30 second practice transcription typing task, followed by 5 transcription typing tasks ( 4 with music, and 1 without music). The experimenter started timing the task when the participant began typing and stopped the participant after 4.5 minutes. Once the participants had finished all 5 typing tasks, they completed demographic questionnaires on paper.

\subsection{Results}

\subsubsection{Typing Speed}

Figure 1 shows a histogram of typing speed for all conditions. Visual inspection suggests the distribution may be bimodal rather than normal with a crossing at around 340 CPM as shown by the overlay which is an approximation of two normal distributions. There may also be 5 outlying data points above 520 CPM.

Scatterplots were created for the Alt Rock and Pop Rock conditions (Figure 2) to compare the speeds achieved by each participant within a single piece of music and establish an appropriate threshold level for separation into 'slow' and 'fast' typist groups. The histogram in 1 suggested a crossing at about 340 CPM. Inspection of the scatterplots led to a refinement of this threshold 


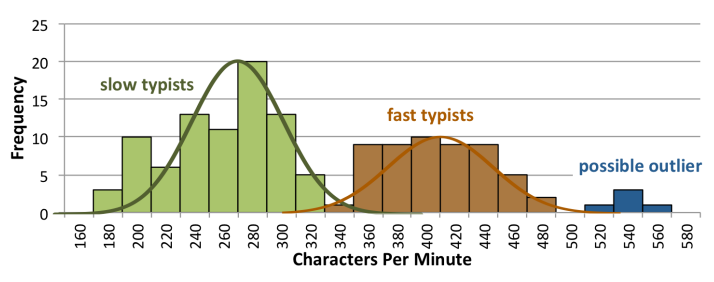

Figure 1: Histogram of CPM showing typist ability grouping with normal distribution overlays and a possible outlier.
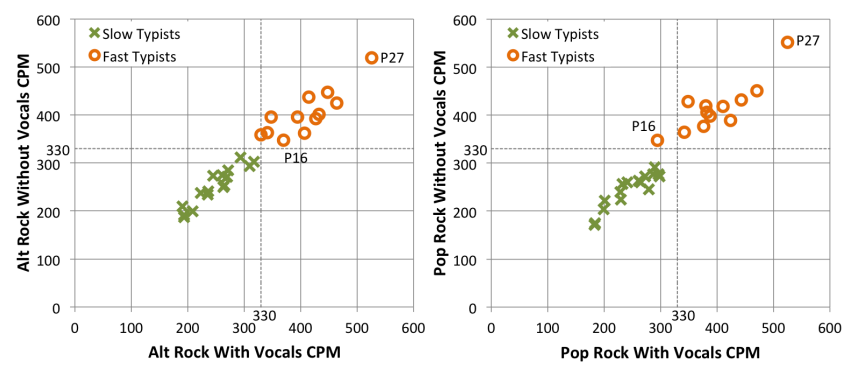

Figure 2: CPM scatterplots for both music styles, showing typist ability classification with the 330 CPM threshold applied. Speeds achieved by participants 16 and 27 are highlighted.

value to $330 \mathrm{CPM}$, resulting in 16 participants classified as slow typists and 12 as fast typists. The only participant that did not easily fit within this classification structure was participant 16 who achieved less than 330 CPM in the Pop Rock Without Vocals condition, but higher than $350 \mathrm{CPM}$ in all other conditions. As $80 \%$ of tasks were completed high above the 330 CPM threshold, participant 16 was classified as a fast typist. The scatterplots also verify that a single participant (P27) achieved over 500 CPM in all tasks.

Repeated-measures ANOVAs were performed on the data using both the 1 by 5 and 2 by 2 experimental design paradigms. A second analysis using mixed designs ANOVAs allowed inclusion of typing ability as a between-participants factor. An alpha level of 0.05 was used in all statistical tests.

All of the underlying assumptions for Repeated Measures ANOVAs were met when the data was treated as a single distribution. The assumption of normality of distribution was assessed for each condition separately using Shaprio-Wilk's test. Despite the bimodal characteristic observed through visual inspection, all of the distributions were found not to deviate significantly from normality. When treated as a single distribution, the Repeated Measures ANOVA resulted in no significant omnibus effects or interactions in the 1 by 5 or 2 by 2 design paradigms.

When typing ability was included in the analysis as a between groups factor a statistical outlier was introduced. The analysis was performed both with and without the outlier included in the dataset. This outlier had no effect on the outcomes using either the 1 by 5 or 2 by 2 analysis paradigms. All other assumptions for Mixed Design ANOVAs were met.

With the 1 by 5 experimental design paradigm the effect of the music was not significant, $\mathrm{F}(4,104)=1.20$, n.s., and neither was the interaction between music and typing ability, $\mathrm{F}(4,104)=1.99$, n.s. Typist ability was a significant between groups factor, $\mathrm{F}(1,26)=90.42, \mathrm{p}<0.001, \eta_{\rho}^{2}=0.78$, with higher speeds achieved by the fast group $(\mathrm{M}=408.17, \mathrm{SD}=53.07)$ than the slow group
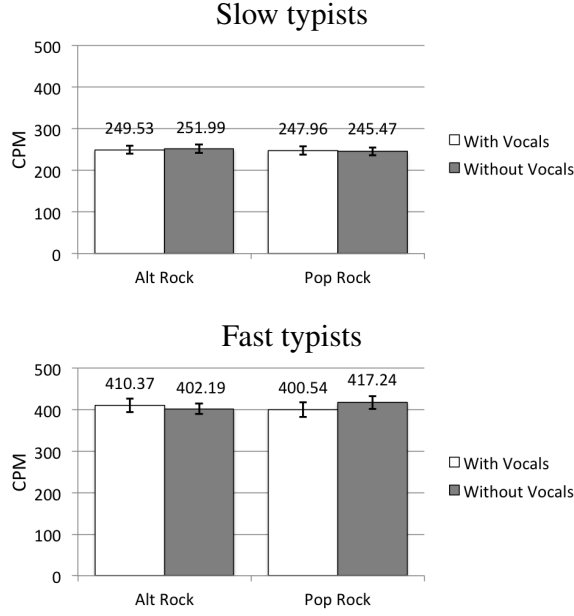

Figure 3: Significant 3-way interaction between music style and typing ability. Error bars show the standard error.

$(M=249.80, S D=39.31)$. This result is expected given the typist ability classification was applied post hoc based on the speeds achieved by each participant.

Using the 2 by 2 analysis paradigm, there were no omnibus effects identified for the vocals condition, $F(1,26)=9.32$, n.s., nor music style, $\mathrm{F}(1,26)=0.06$, n.s. A trend towards a significant interaction between music style and vocals was identified, $\mathrm{F}(1,26)=3.83, \mathrm{p}=0.06, \eta_{\rho}{ }^{2}=0.13$, which suggests any effect of vocals on speed may differ depending on the style of the music, but there is insufficient evidence from this experiment to be sure.

The 3-way interaction between music style, vocals condition and typing ability was significant, $\mathrm{F}(1,26)=8.57, \mathrm{p}=0.007$, $\eta_{\rho}{ }^{2}=0.25$ (Figure 3). A simple 2-way interaction between music style and vocals was significant for fast typists, $\mathrm{F}(1,11)=7.50$, $\mathrm{p}=0.02, \eta_{\rho}^{2}=0.41$, but not for the slow typists, $\mathrm{F}(1,15)=0.77$, n.s. When accompanied by Alt Rock music the fast typists achieved a mean typing speed that was $8 \mathrm{CPM}$ higher with vocals than without vocals (Alt Rock With Vocals: $M=410.37, \mathrm{SD}=56.93$, Alt Rock Without Vocals: $M=402.19, \mathrm{SD}=42.63$ ). For Pop Rock music, a 17 CPM difference in typing speeds was identified between the with and without vocals conditions with higher speeds achieved without vocals (Pop Rock With Vocals: $\mathrm{M}=400.54, \mathrm{SD}=61.86$; Pop Rock Without Vocals: $M=417.24, \mathrm{SD}=52.96)$. However, the simple simple main effect for vocals was not significant for either music style (Alt Rock: $F(1,11)=0.83$, n.s., Pop Rock: $F(1,11)=3.27$ ).

Typist ability was a significant between groups factor, $\mathrm{F}(1,26)=90.28, \mathrm{p}<0.001, \eta_{\rho}{ }^{2}=0.78$, with a lower mean speed achieved by the slow typists than the fast typists (Slow: $M=248.74$, $\mathrm{SD}=39.04$, Fast: $\mathrm{M}=407.48, \mathrm{SD}=53.60$ ).

\subsubsection{Typing Accuracy}

The error rate data had a negative skew so a square root transformation was applied. Before transformation 4 of 5 conditions $(80 \%)$ were strongly non-normal by the Shapiro-Wilk'a test $(\mathrm{p}<.002)$, with the 5 th condition resulting in moderate nonnormality $(\mathrm{p}=0.03)$. After the square root transformation was applied to the data none of the distributions deviated significantly from normality. Figure 4 show a histogram of the transformed 


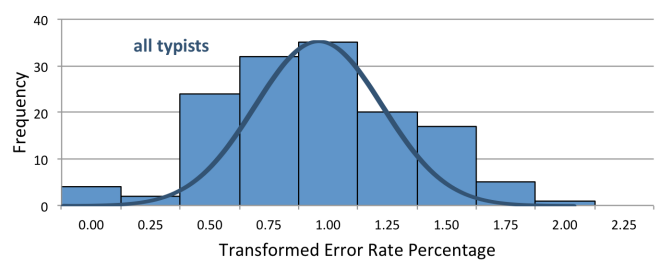

Figure 4: Histogram of Transformed Error Rate Percentage.

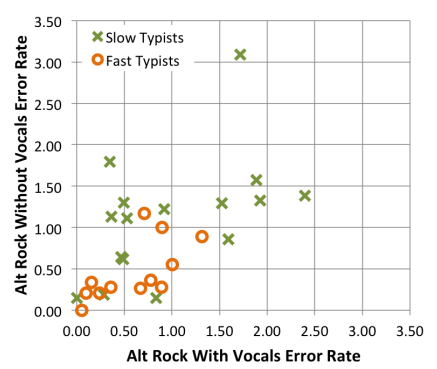

Figure 5: Error Rate scatterplot for Alt Rock music conditions, showing typist ability classification with 330 CPM threshold.

error rate percentage which does not contain the bimodal characteristic observed in the typing speed distribution.

A scatterplot of the two Alt Rock conditions is included in Figure 5 which shows the pattern of error rates achieved by each participant with the slow or fast typist classification. The Pop Rock scatterplot contained similar characteristics. The scatterplot shows that the range of error rates for the slow typists is greater than the for the fast typists group. But, there is a clear mix with some slow typists make few errors, but some fast typists making a comparatively large number.

Treating the transformed error rate percentage data as a single distribution resulted in no significant omnibus effects or interactions when analysed using a repeated measures ANOVA for either paradigm. There were no assumption violations when the dataset was considered as a whole in either the 1 by 5 or 2 by 2 paradigms.

With the typist ability classification applied, two outlying data points are introduced. These two outliers had no impact on the outcomes of the analysis in either paradigm. There were no other violations of assumptions for Mixed Designs ANOVA.

Inclusion of the typist ability classification in the 1 by 5 analysis paradigm resulted in a non-significant omnibus effect for music, $\mathrm{F}(4,104)=0.19$, n.s., and a non-significant interaction between music and typist ability, $\mathrm{F}(4,104)=1.22$, n.s. Typist ability group was a significant between groups factor, $\mathrm{F}(1,26)=6.15, \mathrm{p}=0.02$, $\eta_{\rho}{ }^{2}=0.19$. The mean transformed error rate for the slow typists was higher than for the fast typists (Slow: $\mathrm{M}=0.95 \%, \mathrm{SD}=0.39 \%$, Fast: $\mathrm{M}=0.67 \%, \mathrm{SD}=0.31 \%$ ) meaning that the fast typists made significantly fewer errors.

Performing the analysis using the 2 by 2 design paradigm with typist ability classification resulted in no significant omnibus effects. There was a significant 2-way interaction between vocals and typist ability group, $\mathrm{F}(1,26)=7.24, \mathrm{p}=0.01, \eta_{\rho}{ }^{2}=0.22$. Figure 6 shows that the slow typists made more errors when hearing music that did not contain vocals. In the fast typists group, the magnitude of the difference in transformed error rate percentage between

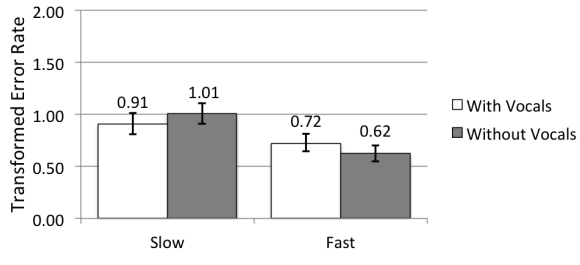

Figure 6: Significant interaction between vocals and typing ability. Error bars show the standard error.

with and without vocals condition is the same as for the slow typists, but in the opposite direction with fewer errors without vocals. A simple main effects analysis for vocals was significant for fast typists, $\left.\mathrm{F}(1,11)=6.92, \mathrm{p}=0.02, \eta_{\rho}{ }^{2}=0.39\right)$ but not for slow typists, $\mathrm{F}(1,15)=0.22$, n.s.

\subsection{Discussion}

The initial aims of this experiment were taken with the assumption that the data collected would be treated as a single distribution. However, when the dataset was analysed as a single distribution for both typing speed and accuracy measures, there were no significant omnibus effects or interactions. Visual inspection of the typing speed distribution suggested a bimodal distribution, leading to a means of classifying participants as either slow or fast and analysing the data to consider whether typist ability matters. The analysis with typist ability classification included led to a number of significant interactions involving this between groups factor. When typist ability classification is applied as a between groups factor the degrees of freedom for the error term are reduced by 1 . This reduction leads to a more appropriate partitioning of the error variance and as the model fits the distribution better the statistical power is increased.

Typing ability grouping was a significant between groups factor for both the typing speed and accuracy measures. The former is expected given that typing speed determined participant allocation to a group. However, although the error rate scatterplots showed a mix of error rates for the slow and fast typists, there was still a significant difference in transformed error rate between the groups with the fast group making fewer errors than the slow group overall. This result is likely to be due to the differences in the spread of error rates by achieved by each group, with a smaller spread for the fast typists than for the slow typists. This outcome indicates that the fast typists were better in both the performance measures, suggesting that they are more skilled typists and not simply faster.

The fast typists made fewer errors when hearing music without vocals. In contrast, the slow typists made more errors without vocals, though the post hoc simple effect analysis was non-significant for the slow group. The non-significant simple effect may be due to the relatively small sample size when the ability classification is applied as a between groups factor. It is possible that the slow typists had to concentrate harder when hearing music that contained vocals, and as a result noticed and corrected their errors more frequently. The differences in effect of vocals on accuracy rates for the slow and fast typists groups warrants further investigation.

Typing ability was not intended to be included as a factor in this first experiment. However, the analysis has shown that this post hoc classification is a very important factor that must be considered in future experiments. 


\section{VOCALS, VOLUME AND TASK DIFFICULTY}

The outcomes from the first experiment showed that the impact of the presence of vocals in a music accompaniment on transcription typing performance is worth pursuing. But, separating the sample into slow and fast ability categories resulted in small group sizes which potentially affected the outcomes. A larger sample of participants is needed to accommodate the ability classification. Sessions in first experiment using a laboratory-approach took 1 hour per participant. To maximise efficiency for the second experiment a classroom-based methodology was used. This approach allowed us to perform an experiment with at least 50 participants in 4 hours.

An online methodology could have been used to get high participation rates in less time with the experiment hosted online and participants completing tasks remotely. This approach would allow us to reach large numbers of participants without the experimenter needing to be present. But, conducting a remote experiment also removes the experimenter's ability to carefully regulate the environment. For example, the experimenter would be unable to tightly control the volume of playback of the music which has previously been shown to affect people (e.g. in [9]). The impact of volume needs to be investigated in a controlled environment prior to any online experiment. So, this experiment includes volume as a between groups IV. If volume does not affect typing performance in a controlled environment then this provides evidence that an online methodology would be appropriate. However, if volume is shown to be a factor that affects performance then careful consideration of how to accommodate the lack of control of volume within the experiment will be needed before any future online approach can take advantage of the large pool of potential participants.

The first experiment also indicated that any effect of the accompanying music differs according to participant skill level. Therefore, it is interesting to investigate whether the effect of accompanying music is consistent across tasks of varying difficulties. When transcription typing in one's own language, performance levels have been shown to be maintained even when words in the presented text are randomly ordered so that the text passage does not have semantic meaning [8]. As such, a simple reordering of the words is unlikely to make the task more difficult and another approach is needed. In this experiment the difficulty of the task is manipulated by including typing tasks in Dutch, which is expected to be a language that than is unknown to the participants. We expect that in harder transcription tasks, the extent of the effect due to the music will be reduced.

\subsection{Method}

The primary aim of this experiment was to investigate whether the presence of vocals in the music affects typing performance and how typist ability interacts with vocals. This experiment also considers if the volume of music affects performance and whether there is any interaction between the volume and presence of vocals. Finally, this experiment looks at whether an increase in task difficulty, manipulated by including a Dutch transcription typing task, reduces the affect of music on typing performance.

\subsubsection{Experimental Design and Hypotheses}

This experiment used a 2 by 2 by 2 mixed design. The between groups IV was music volume (low or high), the within groups IVs were vocals ( 2 levels - with and without vocals) and text ( 2 levels - English and Dutch language). It was expected that typing ability would be included as a between groups factor but this would be incorporated post hoc based on the distributions of speed. The DVs for typing performance were speed measured in CPM, calculated using (1) and error rate calculated using (2).

\subsubsection{Participants and Experimenters}

Both the participants and experimenters were first year undergraduates studying Computer Science. The students were taking a Human Computer Interaction module where they learn how to perform experiments. Within one practical class the students all get to experience being a participant and being an experimenter. Fiftyfive students ( 8 female, 47 male) were participants in this experiment, while another 55 acted as experimenter. All of the participants were aged between 18 and 24. Nine participants were nonnative speakers of English, but all had demonstrated competency in English by achieving International English Language Test (IELT) scores in excess of 6.5. None of the participants in this experiment were familiar with the Dutch language. The participants were not asked to report whether they had a hearing disability or dyslexia as this would have been inappropriate given the classroom context. After the experiment, 5 participants were randomly selected to win $£ 10$ Amazon gift vouchers. The 3 best typists received $£ 30$, $£ 20$ and $£ 10$ Amazon gift vouchers.

\subsubsection{Materials}

The with and without vocals versions of the Alt Rock style music were used in this experiment. The Alt Rock style was chosen over the Pop Rock because the outcomes from the first experiment suggested that vocals might have had a significant effect on performance in the Pop Rock music condition. We did not want to constrain the generalisations of the outcomes from these two experiments to a single style and as there was no clear evidence that vocals had a negative effect with the Alt Rock music, it is more interesting to use this piece as the stimulus.

Four text passages were used for the typing tasks, two in English and two in Dutch. The English text passages again came from The Outlaw of Torn by Edgar Rice Burroughs while the Dutch text passages were taken from Op Eigen Wieken, a Dutch translation of Louisa May Alcott's Good Wives. The Dutch language was chosen for the difficult text condition because the Dutch alphabet is similar to English. Accents were removed from all characters.

Again bespoke webpages hosted the typing tasks. These webpages controlled the music, which began playback when the participant made their first keypress. After 4.5 minutes the webpage generated an alert box to end the task. Volume of playback was also set by the webpage. Half of the participants used webpages which played the music at $100 \%$ volume, while playback was set to $50 \%$ volume for the other participants. The suitability of playback volume (i.e. not too quiet or loud) had previously been verified in a pilot test.

The text passages were counterbalanced so that there were no pairings between the pieces of music and the different texts. The order of experimental conditions was systematically varied to avoid fatigue and practice effects.

All participants completed the typing tasks on personal computers running Windows 7 in the department's software laboratory. The experimenter was instructed to use the FireFox browser and disable spellcheck. Participants were given sets of inexpensive headphones (Astro Tools ATA 1144) to use. 


\subsubsection{Procedure}

The experiment took place during two practical classes for a first year undergraduate module. The students worked in pairs, with one acting as experimenter and the other as the participant. Stepby-step instructions for running the experiment were given to the student acting as experimenter to ensure the procedure was followed correctly, including a script of what to say to the participant. The instructions and process had been thoroughly piloted with pairs of students, including non-native English speakers.

Each experimenter began by explaining the premise of the experiment to their participant. The participants were told to type as naturally as possible without prioritising speed or accuracy. The experimenter then set the computer's volume to maximum and informed consent was taken.

The first typing task allowed both students to practice the process. The music began playing on the participant's first keypress. After 30 seconds an alert message ended the practice task. The first experimental typing task was then loaded into the web browser.

Each of the experimental typing tasks lasted 4.5 minutes. After finishing all 4 typing tasks, participants completed demographic questionnaires. When all the participants had finished the experiment, the first author debriefed the class.

\subsection{Results}

\subsubsection{Typing Speed}

Figure 7 shows a histogram of the CPM data for the high and low volume conditions combined. The crossing point for the two distributions is again at approximately $330 \mathrm{CPM}$. Figure 8 is a scatterplot of CPM values for all participants in both of the English text conditions with classification of typists into fast and slow ability groups using a threshold value of 330 CPM. Visual inspection of the scatterplot verifies a clear gap between the slow and fast typists. The proportion of fast typists in this sample was smaller than in the first experiment, with just 11 out of 55 participants classified as fast typists. Of these 11,6 were in the loud volume condition. Of the 42 slow participants, 22 heard loud music. Participants 14 and 28, both non-native English speakers, were the slowest typists. These participants were removed from the analysis as their Dutch and English typing speeds were similar, implying that both languages were unfamiliar. The other non-native speakers of English achieved lower speeds in Dutch than English, making them suitable for inclusion in the analysis. Participant 54, a non-native English speaker, was the fastest typist in this experiment.

With all the participants considered as a single dataset, 3 of the 8 distributions (37.5\%) were strongly non-normal by a ShapiroWilk's test $(\mathrm{p}<.015)$. But, with participants classified as a fast or slow typist only 1 of the 16 conditions $(6.25 \%)$ had a strong non-normal distribution $(\mathrm{p}=0.015)$. This confirms that, the typing ability classification improves the normality of the distributions. Given the importance of the typing ability classification shown in the first experiment a decision was taken to classify the participants as either fast or slow typists according to their achieved typing speeds in English for the inferential analysis. The data was not analysed as a single distribution in this experiment.

A Mixed Design ANOVA was performed on the CPM data. Volume level (low and high) and typist ability (slow and fast) were between-participants IVs. Presence of vocals (with and without) and language of presented text (English and Dutch) were the within-participant IVs. The assumption of homogeneity of vari-

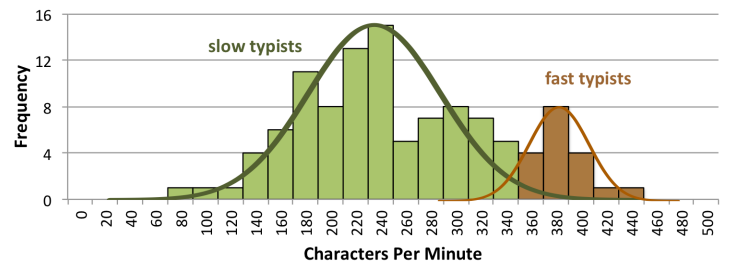

Figure 7: Histogram of CPM showing typist ability grouping with normal distribution overlays.

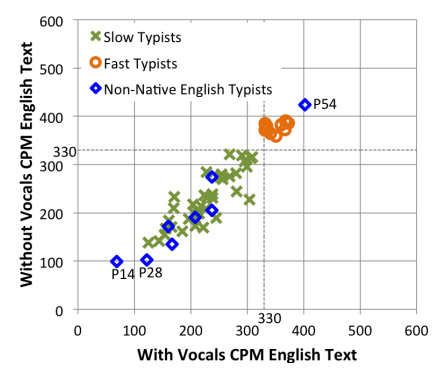

Figure 8: CPM scatterplot of English text condition showing typist ability classification. Non-native English speakers are highlighted, with attention drawn to the speeds achieved by participants 54,14 and 28 .

ance was not met, with violations in both of the English text conditions, (with vocals: $\mathrm{F}(3,49)=3.43, \mathrm{p}=0.02$, without vocals: $\mathrm{F}(3,49)=4.93, \mathrm{p}=0.005)$. As the group sizes are unequal, this assumption violation requires further investigation. When sample sizes are different, heterogeneity of error variance is problematic if the larger variance is associated with the smaller group as the resulting $F$ statistic is inflated leading to a higher chance of a type I error [11]. If the larger variance occurs in the larger group, the F statistic is conservative, with risk of a type II error. Inspection of box plots of each condition showed that smaller variances were associated with the fast typist group which had smaller numbers of participants. As such, the F statistic is at risk of being conservative rather than inflated so the analysis can proceed. Six participants were statistical outliers. The analysis was performed both with and without these outliers included in the dataset and the outcomes were not affected by their inclusion. All other assumptions for Mixed Design ANOVAs were met.

Table 1 presents the outcomes from this analysis. The language of the presented text had a significant omnibus effect with faster speeds achieved when typing in English $(\mathrm{M}=254.16$, $\mathrm{SD}=74.95)$ over Dutch ( $\mathrm{M}=162.60, \mathrm{SD}=47.42)$. There was also a significant omnibus effect for vocals, with higher speeds achieved without vocals $(\mathrm{M}=209.04, \mathrm{SD}=63.50)$ than with vocals $(\mathrm{M}=206.82, \mathrm{SD}=58.86)$. Volume was not identified as an overall significant between groups factor, $F(1,51)=0.32$, n.s., though unsurprisingly the overall effect of typing ability was significant, $\mathrm{F}(1,51)=81.32, \mathrm{p}<0.001, \eta_{\rho}{ }^{2}=0.62$, with higher speeds achieved by those allocated to the fast typists group. The significant twoway interactions are not discussed in detail in this paper as they are subsumed by significant three-way interactions.

The three-way interaction between vocals, volume and typing ability was significant meaning that the two-way interaction between vocals and volume is different across levels of ability. This 


\begin{tabular}{cccc}
\hline Effect & Test & p & $\eta_{\rho}{ }^{2}$ \\
\hline Vocals & $\mathrm{F}(1,49)=7.746$ & 0.008 & 0.137 \\
\hline Vocals x Volume* & $\mathrm{F}(1,49)=4.296$ & 0.043 & 0.081 \\
\hline Vocals x Ability* & $\mathrm{F}(1,49)=8.467$ & 0.005 & 0.147 \\
\hline $\begin{array}{c}\text { Vocals x Volume* } \\
\text { x Ability* }\end{array}$ & $\mathrm{F}(1,49)=6.410$ & 0.015 & 0.116 \\
\hline Text & $\mathrm{F}(1,49)=306.715$ & $<0.001$ & 0.862 \\
\hline Text x Ability* & $\mathrm{F}(1,49)=24.797$ & $<0.001$ & 0.336 \\
\hline Text x Volume* & $\mathrm{F}(1,49)=8.558$ & 0.005 & 0.149 \\
\hline Text x Vocals & $\mathrm{F}(1,49)=3.630$ & \multicolumn{2}{c}{ n.s. } \\
\hline Text x Volume* x Ability* & $\mathrm{F}(1,49)=7.799$ & 0.007 & 0.137 \\
\hline Text x Vocals x Ability* & $\mathrm{F}(1,49)=2.516$ & \multicolumn{2}{c}{ n.s. } \\
\hline Text x Vocals x Volume* & $\mathrm{F}(1,49)=0.093$ & \multicolumn{2}{c}{ n.s. } \\
\hline $\begin{array}{c}\text { Text x Vocals x Volume* } \\
\text { x Ability* }\end{array}$ & $\mathrm{F}(1,49)=0.871$ & \multicolumn{2}{c}{ n.s. } \\
\hline Volume* & $\mathrm{F}(1,49)=0.905$ & \multicolumn{2}{c}{ n.s. } \\
\hline Ability* & $\mathrm{F}(1,49)=90.805$ & $<0.001$ & 0.650 \\
\hline Volume* x Ability* & $\mathrm{F}(1,49)=3.395$ & \multicolumn{2}{c}{ n.s. } \\
\hline
\end{tabular}

Table 1: Summary of Outcomes from 2 by 2 by 2 ANOVA. The * indicates a between groups factor.

three-way interaction is shown in Figure 10. The simple twoway interaction between vocals and volume was significant for fast typists, $\mathrm{F}(1,9)=11.93, \mathrm{p}=0.007, \eta_{\rho}{ }^{2}=0.57$, but not for slow typists, $F(1,40)=0.23$, n.s. For the fast typists, the simple simple main effect of vocals was significant with high volume music, $\mathrm{F}(1,5)=26.68, \mathrm{p}=0.004, \eta_{\rho}{ }^{2}=0.84$, but not with low volume music, $\mathrm{F}(1,4)=0.43$, n.s. When hearing high volume music, the fast typists were slower with music that contained vocals $(M=266.96$, $\mathrm{SD}=96.31)$ than without vocals $(\mathrm{M}=294.50, \mathrm{SD}=101.66)$. The simple simple main effect of volume was significant without vocals, $\mathrm{F}(1,9)=14.47, \mathrm{p}=0.004, \eta_{\rho}{ }^{2}=0.617$, but not with vocals, $\mathrm{F}(1,9)=2.752$, n.s. When hearing music that contained vocals, the fast typists achieved higher speeds in the low volume condition $(\mathrm{M}=311.98, \mathrm{SD}=65.62)$ than in the high volume condition $(M=266.96, S D=96.31)$. These results suggest that for best performance faster typists should avoid listening to music at a loud volume if it contains vocals, but if the volume of the music is lower, the presence of vocals does not have a noticeable effect.

The three-way interaction between text, vocals and typing ability was also significant, indicating that the interaction between text and vocals is different across levels of ability. This three-way interaction is shown in Figure 9. The simple two wayinteraction between text and volume was significant for the fast typists, $\mathrm{F}(1,9)=5.64, \mathrm{p}=0.04, \eta_{\rho}^{2}=0.39$, but not for the slow typists, $F(1,40)=0.03$, n.s. The simple simple main effect of text was significant for both the low, $\mathrm{F}(1,4)=168.64, \mathrm{p}<0.001, \eta_{\rho}{ }^{2}=0.98$, and high volume groups, $\mathrm{F}(1,5)=44.59, \mathrm{p}=0.001, \eta_{\rho}{ }^{2}=0.90$. The fast typists achieved higher speeds at both volume levels when typing in English (Low volume, $\mathrm{M}=365.64, \mathrm{SD}=15.14$; High volume, $\mathrm{M}=368.54, \mathrm{SD}=29.71$ ) over typing in Dutch (Low volume, $\mathrm{M}=261.22, \mathrm{SD}=26.65$; High volume, $\mathrm{M}=192.93, \mathrm{SD}=48.17$ ). The simple simple main effect of volume was significant when typing in Dutch, $\mathrm{F}(1,9)=8.06, \mathrm{p}=0.02, \eta_{\rho}{ }^{2}=0.47$, but not in English,
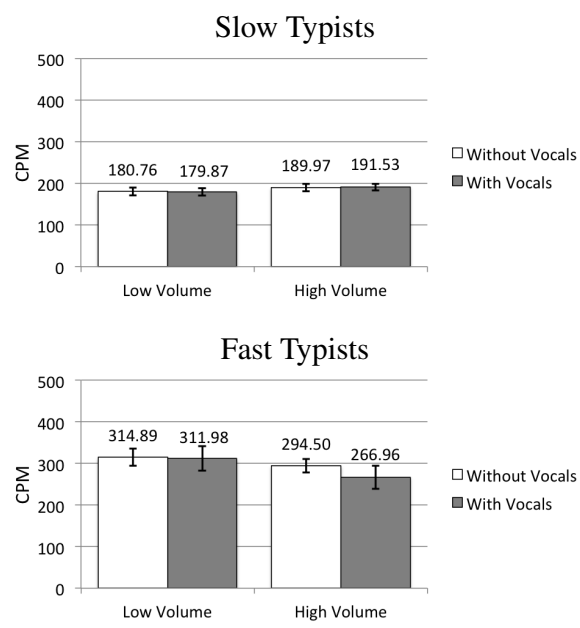

Figure 9: Significant 3-way interaction between volume, vocals and typing ability. Error bars show the standard error.
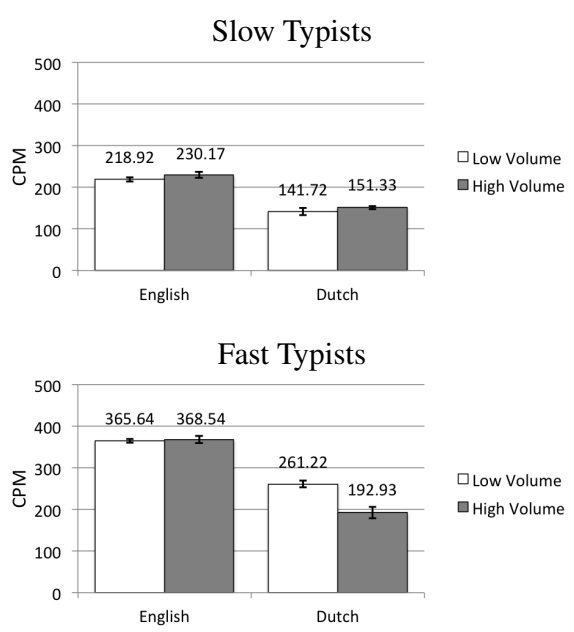

Figure 10: Significant 3-way interaction between text, vocals and typing ability. Error bars show the standard error.

$\mathrm{F}(1,9)=0.06$, n.s. When typing in Dutch, the fast typists achieved higher speeds with low volume music $(\mathrm{M}=261.22, \mathrm{SD}=26.65)$ and slower speeds with high volume music $(\mathrm{M}=192.93, \mathrm{SD}=48.17)$. When typing in a familiar language, the volume of the music did not have an impact on the speeds achieved by the fast typists. However, when typing in an unknown language, the high volume music resulted in lower speeds.

\subsubsection{Typing Accuracy}

As in the first experiment, the error rate data had a strong negative skew. A logarithmic transformation was applied as a square root transformation did not improve the normality of the distributions sufficiently. Before transformation, 12 of 16 conditions $(75 \%)$ were strongly non-normal $(\mathrm{p}<.002)$ by Shapiro-Wilk's. After transformation this reduced to just 3 of 16 conditions (18.75\%) with moderate non-normal distributions ( $\mathrm{p}>.03$ ).

Analysing using a mixed ANOVA on the transformed data as 
a single distribution resulted in a significant omnibus effect for text, $\mathrm{F}(1,51)=6.00, \mathrm{p}=0.02, \eta_{\rho}{ }^{2}=0.11$. The transformed error rate was higher with Dutch $(\mathrm{M}=0.23 \%, \mathrm{SD}=0.59 \%)$ than with English text $(\mathrm{M}=0.05 \%, \mathrm{SD}=0.64 \%)$. There was a significant omnibus effect for vocals, $\mathrm{F}(1,51)=5.64, \mathrm{p}=0.02, \eta_{\rho}{ }^{2}=0.10$, with higher error rates with vocals $(\mathrm{M}=0.24 \%, \mathrm{SD}=0.67 \%)$ than without vocals $(\mathrm{M}=0.07 \%, \mathrm{SD}=0.60 \%)$. Volume was not a significant factor, $\mathrm{F}(1,51)=0.36$, n.s., and there were no significant interactions.

\subsection{Discussion}

In this experiment, when transcription typing while listening to music that contained vocals the participants typed significantly slower with higher error rates than when accompanied by instrumental music. This higher performance without vocals indicates that the typing task was easier with instrumental music and suggests that if task performance is important, people should choose to listen to instrumental music over music containing vocals.

Although there was a significant omnibus effect for vocals, the interaction between vocals, volume and typist ability is particularly interesting as for the slow typists the omnibus effect of vocals is not evident. Even with comparatively small numbers of fast typists in the experiment, for whom the effect of vocals is clear with the high volume music, the omnibus effect for vocals is achieved which demonstrates the strength of the effect for the fast typists. These experiments are quite different in nature and objective to Shaffer's [7] work, but the outcomes do seem to contradict the assertion that skilled typists are not affected by concurrent verbal material. This could be due to the differences between hearing spoken words and listening to music containing vocals, but further investigation is needed.

The other significant three-way interaction between volume, text and typing ability is also interesting as it clearly shows the effect of the background music on typing speed is connected to the difficulty of the task, but not in the way that we initially proposed. When typing in English, a task that was comparatively easy for all participants, the volume of the music had no obvious effect. However, when typing in Dutch, the fast typists group achieved significantly lower speeds when accompanied by louder music. This suggests that when the skilled participants had to concentrate more on the task, the louder music was more distracting and reduced their performance. For simple tasks, we might conclude that volume does not have a big effect but in more complex situations, the volume of the music should be carefully considered.

In this experiment, the participants heard music in all of the experimental conditions. There was no condition where the participants typed without music and so their base typing performance level was not included within the analysis. This limitation was caused primarily by the setting of the experiment, i.e. as part of a practical class rather than using a laboratory. The classroom is a busy environment, which is typically quiet, but not silent. It would have been hard to control the room sufficiently to eliminate confounds for a no-music IV so one was not included.

The modest number of fast typists in the experiment is a further limitation. For the accuracy DV, speed classification did not factor in the analysis so it is not an issue. But, for the speed analysis only 11 of 53 typists were classified as fast, limiting the generalisability of the outcomes. Due to the setting of the experiment, it was not possible to select participants with a range of abilities and no further participants could be added without introducing confounds. Experiments with a large group of fast typists are needed to validate the typing speed outcomes.

\section{CONCLUSIONS}

This paper has highlighted the importance of the differences between skilled and novice typists. The typing literature, much of which was written in the 1980 s, has typically focused on working with typists that had been trained. But, these two experiments have shown that the impact of different dimensions of background music on typing performance is dependent on typing skill level.

Both experiments in this paper have also shown that vocals can have a negative affect on transcription typing performance. Although this outcome may seem obvious, these experiments provide empirical evidence that was previously missing from the literature. The second experiment demonstrated that high volume music can have a negative affect on typing speed, so we recommend that careful consideration of the researchers' inability to control volume be taken in any future experiments performed online. This result also indicates that different dimensions of music have different effects, and suggests that we might be able to exploit loud, instrumental music to improve performance when working at a computer, especially for tasks where the user's level of skill is high. But, for difficult tasks, skilled user's should choose low volume music to maintain high levels of performance.

\section{REFERENCES}

[1] Heather McElrea and Lionel Standing. Fast Music Causes Fast Drinking. Perceptual and Motor Skills 75, 1992, 362 - 362. Issue 2.

[2] Ronald E. Milliman. Using Background Music to Affect the Behavior of Supermarket Shoppers. Journal of Marketing 46, 3, 1982, $86-91$.

[3] Teresa Lesiuk. The effect of music listening on work performance. Psychology of Music 33, 2, 2005,173 - 191.

[4] Kevin Taylor. 2007. An analysis of computer use across 95 organisations in Europe, North America and Australasia. Technical Report. Wellnomics Ltd.

[5] K. Kallinen. Reading news from a pocket computer in a distracting environment: Effects of tempo of background music. Computers in Human Behavior, 18, 2002, 537 - 551.

[6] M. B. Jensen. The influence of jazz and dirge music upon speed and accuracy of typing. Journal of Educational Psychology. 22, 1937, 458 - 462. Issue 6.

[7] L. H. Shaffer. 1975. Multiple attention in continuous verbal tasks. In Attention and Performance V, P. M. A. rabbit and S. Dornic (Eds.). New York: Academic Press, 157 - 167.

[8] Timothy A. Salthouse. Perceptual, Cognitive, and Motoric Aspects of Transcription Typing. Psychological Bulletin 99, 1986, 303 - 319. Issue 3.

[9] J. Edworthy and H Waring. The effects of music tempo and loudness level on treadmill exercise. Ergonomics 49, 15, 2006, $1597-1610$.

[10] L. J. West and Y. Sabban. Hierarchy of stroking habits at the typewriter. Journal of Applied Psychology 67, 1982, 370 376.

[11] Barbara G Tabachnick and Linda S Fidell. 2007. Experimental Designs Using ANOVA. Thomson/Brooks/Cole. 